\title{
Associação entre estilo de vida e percepção de saúde em estudantes universitários
}

\section{Association Between Lifestyle and Health Perception in College Students}

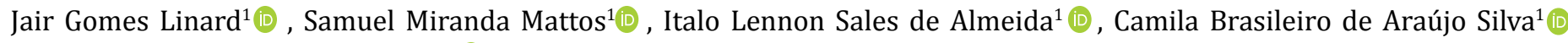 \\ Thereza Maria Magalhães Moreira ${ }^{2,3}$ (D)
}

1. Discente do Programa de Pós-Graduação em Saúde Coletiva pela Universidade Estadual do Ceará (UECE), Fortaleza, CE, Brasil. 2. Docente do Programa de Pós-Graduação em Saúde Coletiva pela Universidade Estadual do Ceará (UECE), Fortaleza, CE, Brasil. 3. Docente do Programa de Pós-Graduação em Cuidados Clínicos em Enfermagem e Saúde, Fortaleza, CE, Brasil.

\begin{abstract}
Resumo
Objetivo: analisar os aspectos sociodemográficos, o estilo de vida de estudantes universitários e sua associação com a autopercepção de saúde dos estudantes universitários. Métodos: trata-se de um estudo transversal analítico realizado com 119 universitários. Os dados foram coletados por meio do questionário "Indicadores de Saúde e Qualidade de Vida de Acadêmicos (Isaq-A)" e analisados no programa estatístico SPSS 20.0. Para análise descritiva, foram calculadas as frequências simples e relativas das variáveis do estudo e medidas de tendência central e de dispersão, também se utilizou estatística bivariada. Foi realizada a análise com modelo de regressão logística hierarquizada, no qual as variáveis foram divididas em variáveis sociodemográficas e de estilo de vida. 0 desfecho adotado foi o índice de qualificação discente (IQD). 0 estudo foi aprovado no Comitê de Ética sob o Parecer no 1.582 .378 . Resultados: na análise bivariada houve associação estatisticamente significativa com sexo $(p=0,03)$; nível de estresse $(p=0,004)$; sentir-se satisfeito com a vida $(\mathrm{p}=0,001)$ e consumo de álcool (últimos 30 dias) $(\mathrm{p}=0,002)$. Conclusão: ao longo do percurso acadêmico, o estilo de vida sofre alterações negativas e positivas.
\end{abstract}

Palavras-chave: Estilo de vida. Estudante. Percepção.

\begin{abstract}
Objective: to analyze the sociodemographic aspects and lifestyle of college students and their association with self-rated health of college students. Methods: this is an analytical cross-sectional study conducted with 119 university students. Data were collected through the questionnaire "Health Indicators and Quality of Life of Academics (Isaq-A)" and analyzed using the SPSS 20.0 statistical program. For descriptive analysis, the simple and relative frequencies of the study variables were calculated and measures of central tendency and dispersion, also used bivariate statistics. Analysis was performed using a hierarchical logistic regression model, in which the variables were divided into sociodemographic and lifestyle variables. The outcome adopted was the student qualification index (IQD). The study was approved by the Ethics Committee under opinion No. 1,582,378. Results: showed in the bivariate analysis statistically significant association with gender $(p=0.03)$; stress level $(p=0.004)$; feeling satisfied with life $(p=0.001)$ and alcohol consumption (last 30 days) $(p=0.002)$. Conclusion: along the academic course, the lifestyle undergoes negative and positive changes.
\end{abstract}

Key words: Lifestyle. Student. Perception.

\section{INTRODUÇÃO}

A população universitária cresceu, significativamente, nas últimas décadas. Percebe-se que, entre 2007 e 2017, o número de ingressantes na educação superior aumentou $56,4 \%$ no total de matrículas da rede pública e privada no ensino superior ${ }^{1}$.

O período de transição da adolescência para a vida adulta é uma fase crítica, marcada por mudanças e novos desafios. Nessa fase, permeiam, nos cotidianos desses jovens, novos acontecimentos, costumes, hábitos, mudanças de ordem comportamental e social. Atividades ocupacionais, laborais, ingresso no meio universitário, inserção no mercado de trabalho, novas relações interpessoais, relacionamentos afetivos, construção familiar, entre outras, estão fortemente inseridas na vida dos jovens ${ }^{2,3,4,5}$. Com esse crescente aumento entre os universitários, o estilo de vida tem sofrido significativas mudanças, pois, nessa fase, há mudanças nas rotinas desses jovens e hábitos de saúde que passam a explicar ocorrência de agravos à saúde, nos quais o comportamento do indivíduo tem papel importante ${ }^{2,3}$. $\mathrm{O}$ ingresso no ensino superior é caracterizado por profundas mudanças de ordem social e comportamental, as quais podem levar a alterações em seus estilos de vida ${ }^{4,5}$.

A aquisição de hábitos saudáveis como prática de atividade física, alimentação balanceada, comportamento preventivo e melhoria do sono podem reduzir os fatores de riscos para o desenvolvimento de doenças cardiovasculares, sendo, portanto, ações de baixo custo, quando comparadas com os ascendentes orçamentos dos tratamentos medicamentosos e dependentes 
de alta tecnologia2,3,4.

Em contrapartida, desigualdades sociais, insegurança, comportamentos sedentários, inatividade física, alimentação não saudável e o estresse configuram-se estilos de vida não saudáveis, colocando a saúde em risco ${ }^{5,6}$.

Outro fator prejudicial à saúde é o tabagismo, sendo a principal causa de mortalidade evitável nos jovens ${ }^{7,8}$. Estudos têm demonstrado que, ao ingressar no ensino superior, muitos jovens adquirem o hábito de fumar, reduzem o nível de atividade física e tendem a ganhar peso com o avançar dos anos acadêmicos ${ }^{7,8}$. O consumo de bebidas alcoólicas está presente, precocemente, na vida dos jovens, tendo seu início por volta dos 17 anos, idade que pode coincidir com ingresso no ensino superior ${ }^{9,10}$.

A razão pela qual alguns jovens seguem estilos de vida mais saudáveis e outros menos não está completamente elucidada. Percebe-se que os universitários, inclusive os pertencentes à área da saúde, são vulneráveis a ações danosas à saúde, como o consumo de álcool, o uso de tabaco e a inadequação alimentar, o que não era de se esperar, visto que, em seu período acadêmico, difundem-se o cuidado e a promoção da saúde s,10,11. $^{9,}$

Com isso, ações interventivas devem ocorrer no sentido de sensibilizar esse público para o enfrentamento aos comportamentos nocivos à saúde e a adesão ao estilo de vida saudável durante o percurso acadêmico. Diante do exposto, o objetivo deste artigo foi analisar os aspectos sociodemográficos e o estilo de vida de estudantes universitários e a sua associação com a autopercepção de saúde em estudantes de uma universidade pública do Nordeste brasileiro.

\section{MÉTOdOS}

Estudo transversal, quantitativo, realizado em uma universidade pública do município de Fortaleza-Ceará-Brasil, no ano de 2016. A população do estudo foi constituída por estudantes da Universidade Estadual do Ceará (UECE), ingressantes e concludentes do curso de Educação Física regularmente matriculados.

Recorremos a uma amostra não probabilística, com uma população composta por 65 estudantes ingressantes e 70 estudantes concludentes, totalizando 135 alunos do curso de educação física. Ao findar o período da coleta de dados, a amostra obtida para o presente estudo foi de 59 estudantes ingressantes e 60 concludentes. 0 alcance amostral foi de $88 \%$ da população.

Foram excluídos da amostra os estudantes que apresentaram deficiência física, pois não havia meios disponíveis para a realização de medidas antropométricas para esse grupo. Quando ocorreram tais casos, novos participantes foram incluídos.

Consistiram estudantes ingressantes aqueles que adentraram na universidade no período de 2015.2 e 2016.1, (primeiro ano do curso). Os concludentes foram aqueles que estiveram matriculados nas disciplinas de monografia I e II que integram a matriz curricular do curso de educação física e são ofertadas no sétimo e oitavo semestre (último ano), período em que os alunos se aproximam da conclusão do seu curso de graduação. A coleta de dados deu-se por meio da aplicação do questionário Indicadores de Saúde e Qualidade de Vida de Acadêmicos (Isaq-A). Esse questionário contém informações sobre os domínios: sociodemográficos, estilo de vida e indicadores do ambiente e condições de aprendizagem 12. Além das variáveis contidas no questionário, foram coletadas medidas antropométricas (peso e altura).

A percepção de saúde foi avaliada pela indagação: de um modo geral, como você considera o seu estado de saúde atual? As opções de resposta eram as seguintes: muito ruim, ruim, regular, bom e muito bom. As variáveis independentes foram agrupadas em sociodemográficas e indicadores do estilo de vida.

Foram calculadas as medidas estatísticas descritivas médias e o desvio-padrão das variáveis quantitativas. Em seguida, foi realizada estatística analítica bivariada e multivariada para o entrecruzamento das diversas variáveis, com utilização dos testes estatísticos conforme tipo de variável.

Para variáveis categóricas, foi adotado o teste Qui-quadrado de Pearson, quando categóricas dicotômicas ou para numérica paramétrica, utilizamos o T de Student e para amostras independentes ou numérica não paramétrica, o teste de $U$ de Mann-Whitney. Para estimar a força de associação entre as características sociodemográficas e o estilo de vida e a autopercepção de saúde, foi calculada a odds ratio (OR), com intervalo de confiança de $95 \%$.

Os procedimentos de coleta de dados foram iniciados após os esclarecimentos sobre a pesquisa, a assinatura do Termo de Consentimento Livre e Esclarecido. Essa pesquisa foi aprovada pelo Comitê de Ética em Pesquisa da Universidade da Integração Lusofonia Afro-Brasileira (UNILAB) no Parecer de número 1.582.378.

\section{RESULTADOS}

Participaram do estudo 119 discentes. A maioria do sexo masculino, com até 24 anos. A idade variou de 17 a 48 anos, com média de $22,84 \pm 4,8$ anos. $89,1 \%$ não possuíam companheiro; $54,6 \%$ exerciam atividade remunerada, com carga horária de trabalho semanal, variando de zero a 54 horas, com mediana de 12 horas.

O local de residência de $89,1 \%$ foi fortaleza, uma vez que $79,8 \%$ residiam com os pais. 0 número máximo de pessoas na residência foi de 8 , com média de $3,5 \pm 1,2$ pessoas. Tanto a escolaridade do pai quanto da mãe foi acima de oito anos de estudo $68,1 \%$ e $73,1 \%$, respectivamente. Esses dados podem ser visualizados na tabela 1 . 
Tabela 1. Distribuição das características sociodemográficas dos estudantes do primeiro e último ano do curso de graduação em educação física. Fortaleza-Ceará-Brasil, 2017.

\begin{tabular}{|c|c|c|}
\hline Características sociodemográficas & f & $\%$ \\
\hline \multicolumn{3}{|l|}{ Sexo } \\
\hline Masculino & 78 & 65,5 \\
\hline Feminino & 41 & 34,5 \\
\hline \multicolumn{3}{|l|}{ Faixa etária } \\
\hline Até 24 anos & 87 & 73,1 \\
\hline Acima de 24 anos & 32 & 26,9 \\
\hline \multicolumn{3}{|l|}{ Situação conjugal } \\
\hline Sem companheiro & 106 & 89,1 \\
\hline Com companheiro & 13 & 10,9 \\
\hline \multicolumn{3}{|l|}{ Exerce atividade remunerada } \\
\hline Sim & 65 & 54,6 \\
\hline Não & 54 & 45,4 \\
\hline \multicolumn{3}{|l|}{ Carga horária de trabalho semanal } \\
\hline Até 30 horas semanais & 103 & 86,6 \\
\hline Acima de 30 horas semanais & 16 & 13,4 \\
\hline \multicolumn{3}{|l|}{ Renda } \\
\hline Com renda & 65 & 54,6 \\
\hline Sem renda & 54 & 45,4 \\
\hline \multicolumn{3}{|l|}{ Local de residência } \\
\hline Fortaleza & 106 & 89,1 \\
\hline Região metropolitana & 13 & 10,9 \\
\hline \multicolumn{3}{|l|}{ Reside com os pais } \\
\hline $\operatorname{Sim}$ & 95 & 79,8 \\
\hline Não & 24 & 20,2 \\
\hline \multicolumn{3}{|l|}{ Escolaridade do pai } \\
\hline Até 8 anos de estudo & 38 & 31,9 \\
\hline Acima de 8 anos de estudo & 81 & 68,1 \\
\hline \multicolumn{3}{|l|}{ Escolaridade da mãe } \\
\hline Até 8 anos de estudo & 32 & 26,9 \\
\hline Acima de 8 anos de estudo & 87 & 73,1 \\
\hline
\end{tabular}

Fonte: o(s) autor(es), 2019.

No tocante aos indicadores do estilo de vida e saúde dos discentes (Tabela 2), 70,6\% referiram apresentar estresse, $89,1 \%$ relataram dormir bem e $47,9 \%$ afirmaram que se sentiam satisfeitos com a vida. O IMC médio foi de $24,6 \pm 3,8 \mathrm{~kg} / \mathrm{m}^{2}$, sendo que $57,1 \%$ estavam com baixo peso/normal. O tempo de atividade física por semana em minutos variou de 0 a 270 , com mediana de 60 minutos, 96,6\% realizavam até 150 minutos de atividade física por semana. Dessa forma, $84,9 \%$ dos discentes foram considerados ativos fisicamente.

A maioria dos discentes referiram não possuir pressão alta $95,8 \%$, colesterol alto $92,4 \%$ e diabetes $100 \%$. Os estudantes
97,5\% não tinham hábito de fumar, 70,5\% afirmaram não consumir álcool semanalmente, e $63 \%$ relataram não consumir álcool nos últimos 30 dias. Apenas 3,4\% discentes admitiram ter feito uso de maconha nos últimos 30 dias, e 2,5\% fizeram uso de anabolizantes no mesmo período, sendo a prevalência da percepção de saúde positiva 76,4\%.

A percepção de vida positiva se mostrou associada ao sexo $(p=0,03)$, nível de estresse $(p=0,004)$, sentir-se satisfeito com a vida ( $p<0,001)$ e consumo de álcool (últimos 30 dias) $(p=0,002)$, conforme descrito na tabela 3. 
377 Estilo de vida e percepção de saúde de universitários

Tabela 2. Distribuição dos indicadores do estilo de vida e saúde dos estudantes participantes da pesquisa. Fortaleza-Ceará-Brasil, 2017.

\begin{tabular}{|c|c|c|}
\hline Indicadores do estilo de vida e saúde & $f$ & $\%$ \\
\hline \multicolumn{3}{|l|}{ Nível de estresse } \\
\hline Nunca/raramente & 35 & 29,4 \\
\hline Às vezes/quase sempre/sempre & 84 & 70,6 \\
\hline \multicolumn{3}{|l|}{ Com que frequência dorme bem } \\
\hline Nunca/raramente & 13 & 10,9 \\
\hline Às vezes/quase sempre/sempre & 106 & 89,1 \\
\hline \multicolumn{3}{|l|}{ Satisfação com a vida } \\
\hline Não está satisfeito & 06 & 5,0 \\
\hline Mais ou menos satisfeito & 53 & 44,5 \\
\hline Está satisfeito & 57 & 47,9 \\
\hline Não sabe informar & 02 & 1,7 \\
\hline \multicolumn{3}{|l|}{ Índice de Massa Corporal } \\
\hline Baixo peso/peso normal & 68 & 57,1 \\
\hline Sobrepeso/obesidade & 51 & 42,9 \\
\hline \multicolumn{3}{|c|}{ Tempo de atividade física por semana (em minutos) } \\
\hline Até 150 min/semana & 115 & 96,6 \\
\hline Acima de $150 \mathrm{~min} / \mathrm{semana}$ & 04 & 3,4 \\
\hline \multicolumn{3}{|l|}{ Classificação do nível de atividade física } \\
\hline Ativos fisicamente & 101 & 84,9 \\
\hline Insuficientemente ativos ou inativos & 18 & 15,1 \\
\hline \multicolumn{3}{|l|}{ Pressão alta autorreferida } \\
\hline Sim & 05 & 4,2 \\
\hline Não & 114 & 95,8 \\
\hline \multicolumn{3}{|l|}{ Colesterol alto autorreferido } \\
\hline $\operatorname{Sim}$ & 09 & 7,6 \\
\hline Não & 110 & 92,4 \\
\hline \multicolumn{3}{|l|}{ Diabetes autorreferido } \\
\hline Sim & 0 & - \\
\hline Não & 119 & 100,0 \\
\hline \multicolumn{3}{|l|}{ Tabagismo } \\
\hline $\operatorname{Sim}$ & 03 & 2,5 \\
\hline Não & 116 & 97,5 \\
\hline \multicolumn{3}{|l|}{ Consumo de álcool (semanalmente) } \\
\hline Sim & 35 & 29,5 \\
\hline Não & 84 & 70,5 \\
\hline \multicolumn{3}{|l|}{ Consumo de álcool (nos últimos 30 dias) } \\
\hline Sim & 44 & 37,0 \\
\hline Não & 75 & 63,0 \\
\hline \multicolumn{3}{|l|}{ Uso de maconha (nos últimos 30 dias) } \\
\hline $\operatorname{Sim}$ & 04 & 3,4 \\
\hline Não & 115 & 96,6 \\
\hline
\end{tabular}




\begin{tabular}{lrr}
\hline Indicadores do estilo de vida e saúde & f & $\%$ \\
\hline Uso de anabolizantes (nos últimos 30 dias) & & 2,5 \\
$\quad$ Sim & 03 & 97,5 \\
$\quad$ Não & 116 & 76,4 \\
Percepção de saúde dos discentes participantes & & 23,6 \\
Positiva & 91 & 28 \\
Negativa & & \\
\hline
\end{tabular}

Fonte: o(s) autor(es), 2019.

Tabela 3. Associação entre características sociodemográficas, estilo de vida e autopercepção de saúde entre os discentes participantes do estudo. Fortaleza-Ceará-Brasil, 2017.

\section{Autopercepção de saúde}

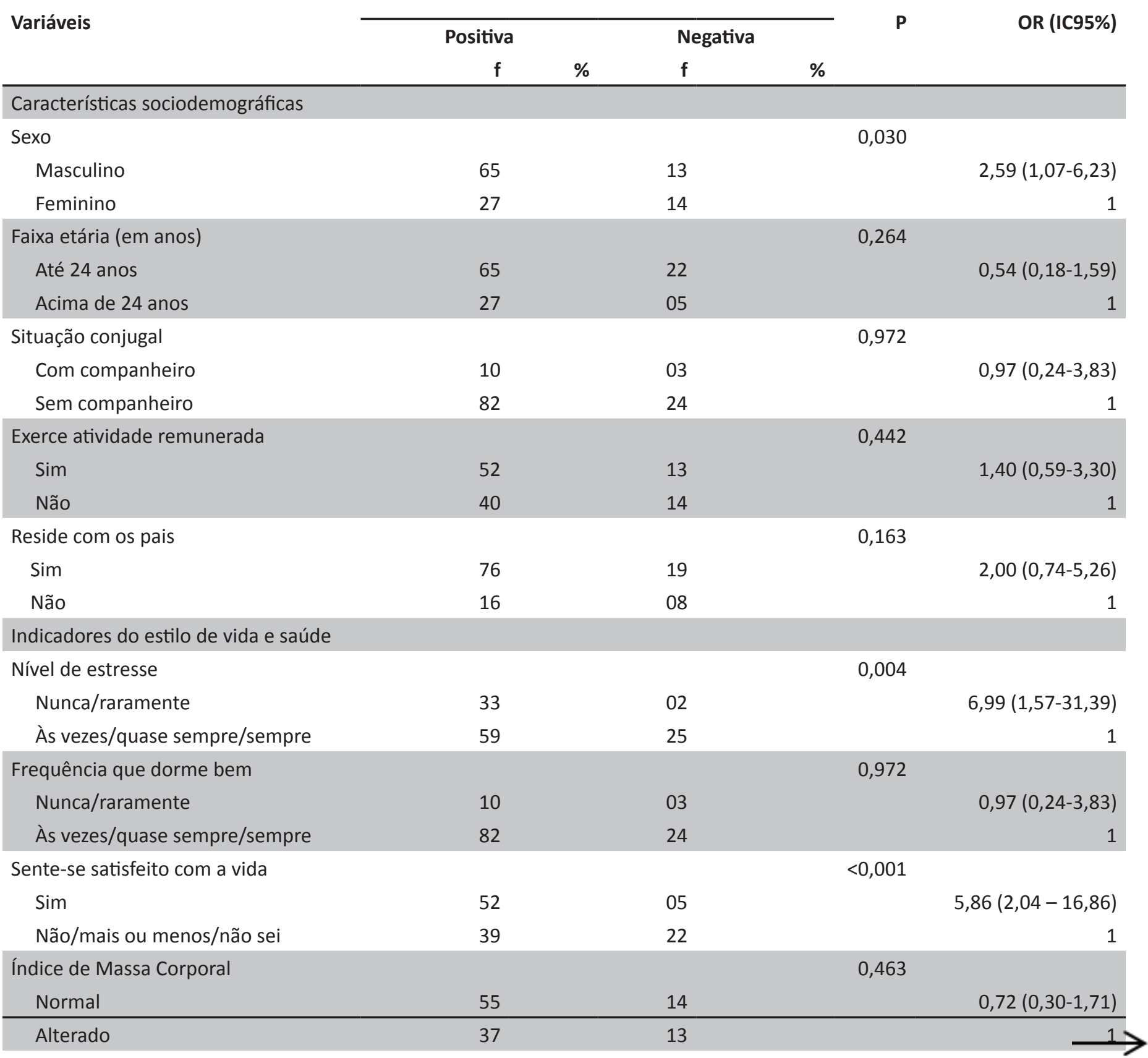




\section{Autopercepção de saúde}

\begin{tabular}{|c|c|c|c|c|c|c|}
\hline \multirow{2}{*}{ Variáveis } & \multicolumn{2}{|l|}{ Positiva } & & & \multirow[t]{2}{*}{$\mathbf{P}$} & \multirow[t]{2}{*}{ OR (IC95\%) } \\
\hline & $f$ & $\%$ & f & $\%$ & & \\
\hline Nível de atividade física & & & & & 0,242 & \\
\hline Ativos fisicamente & 80 & & 21 & & & $1,90(0,63-5,67)$ \\
\hline Insuficientemente ativos ou inativos & 12 & & 06 & & & 1 \\
\hline Pressão alta autorreferida & & & & & 0,883 & \\
\hline Sim & 04 & & 01 & & & $1,18(0,12-11,04)$ \\
\hline Não & 88 & & 27 & & & 1 \\
\hline Colesterol alto autorreferido & & & & & 0,105 & \\
\hline Sim & 05 & & 04 & & & $0,33(0,82-1,33)$ \\
\hline Não & 87 & & 23 & & & 1 \\
\hline Tabagismo & & & & & 0,656 & \\
\hline Sim & 02 & & 01 & & & $0,57(0,05-6,62)$ \\
\hline Não & 90 & & 26 & & & 1 \\
\hline Consumo de álcool (últimos 30 dias) & & & & & 0,994 & \\
\hline Sim & 34 & & 10 & & 0,002 & $0,99(0,41-2,42)$ \\
\hline Não & 58 & & 17 & & & 1 \\
\hline
\end{tabular}

Fonte: o(s) autor(es), 2019.

Conforme foi apresentado, há maior probabilidade de um universitário apresentar percepção de vida positiva se for do sexo masculino (OR=2,59; IC 95\% 1,07-6,23), nunca ou raramente sentir-se estressado (OR=6,99; IC95\% 1,57-31,39) e apresentar satisfação com a vida (OR= 5,86; IC95\% 2,04-16,86).

A prevalência global da percepção de saúde positiva foi de $76,4 \%$, sendo maior entre os estudantes, ser do sexo masculino, com até 24 anos, sem companheiro, que exercer atividade remunerada e residem com os pais quando comparados aos seus opostos. Com relação aos indicadores do estilo de vida nível de estresse e dormir bem às vezes/quase sempre/sempre, satisfação com a vida, índice de massa corporal normal, ativos fisicamente, não consumo de álcool nos (último 30 dias), não diabetes, não colesterol elevado e não pressão elevada autoreferido também apresentaram maiores prevalências de percepção de saúde positiva.

\section{DISCUSSÃO}

O objetivo do estudo foi analisar os aspectos sociodemográficos e o estilo de vida de estudantes universitários e sua associação com a autopercepção em saúde. Os resultados indicaram que a percepção de saúde apresentou associação com o sexo, o nível de estresse, satisfação com a vida e consumo de álcool, corroborando os achados de outras populações $2,3,4,5,6,7,8,13$.

Embora tenha tratado de estudantes da área da saúde, esse público requer atenção nas investigações, visto que a relação conhecimento e adoção de comportamentos saudáveis não está completamente elucidada ${ }^{13}$.

Em relação ao sexo, ficou evidenciado que os homens têm maior probabilidade de ter percepção positiva de saúde em comparação às mulheres. Colaborando com os achados, um estudo realizado na região Noroeste do Ceará, em diversos cursos de graduação, apresentou associação positiva da percepção de saúde em ambos os sexos ${ }^{14}$.

O estresse pode ser um fator prejudicial à saúde. Em geral, estudos relatam que pessoas estressadas possuem maior risco de desenvolver transtornos mentais e doenças 15 . Em relação aos universitários, esse fator aumenta, devido às cobranças de trabalhos, disciplinas, produção acadêmica, entre outras tarefas ${ }^{15,16}$. A prática regular de atividade física e/ou práticas integrativas e complementares (Yoga, Reiki, Biodança, Aromoterapia,...), aliada com a alimentação saudável e com o comportamento preventivo são estratégias para diminuição do estresse $^{15,16}$.

A entrada no ensino superior para os jovens vem acarretada de novos desafios ${ }^{13}$. Estar satisfeito com a vida, mantendo o equilíbrio físico e emocional é essencial para uma formação de qualidade. Estudos mostram que os alunos que têm um ambiente agradável, prazeroso e estruturado conseguem atingir melhor nível de assimilação e aprendizado ${ }^{15}$. Entretanto, pesquisas demonstram que estudantes da saúde têm sua qualidade de vida afetada, negativamente, devido a altas cobranças de atividades referentes à formação ${ }^{16}$. 
Outro fator são as relações interpessoais. No contexto universitário, os jovens tendem a vivenciar novas experiências, nem sempre benéficas à saúde. A inserção em novos grupos de amizades e relacionamentos, por vezes, perpassa a novas experiências, entre elas, a ingestão de álcool e o uso de substâncias ilícitas.

O consumo e a utilização de álcool e drogas no ensino superior têm crescido nos últimos anos. Em estudo no Nordeste brasileiro, identificou que 70,18\% já usaram álcool em algum momento da vida ${ }^{17}$. Quando analisada, a região Sudeste teve prevalência $79,8 \%{ }^{18}$. Pesquisa nacional apontou que mais de $75 \%$ no Brasil ingeriu algum tipo de bebida alcoólica ${ }^{19}$.

É preocupante a alta prevalência em relação à utilização do álcool nos estudantes da área da saúde, como Educação Física, Nutrição, Ciências Biológicas, Medicina, Odontologia, Enfermagem, Fitoterapia, entre outras, por entender que serão futuros profissionais e formadores de opinião sobre saúde na sociedade ${ }^{18}$. Esses comportamentos merecem atenção, pois sua prática, em longo prazo, trará prejuízos à saúde ${ }^{20}$.

Em relação à limitação do estudo, tem-se o fato de ser um delineamento transversal, que avaliou a associação do estilo de vida e a percepção de saúde de um curso de graduação em uma universidade de Fortaleza. Sendo assim, os resultados dessa pesquisa não devem ser generalizados para todos os estudantes universitários; entretanto, eles auxiliarão a compreender o perfil da amostra representada e em ações de promoção da saúde no cenário universitário. Além disso, o estudo colabora com a prática profissional dos estudantes, pois atitudes adquiridas, antes e durante a graduação, podem ser levadas para a vida profissional. Por isso, é relevante ter um estilo de vida ativo e saudável.

\section{CONCLUSÃO}

A associação do estilo de vida e percepção de saúde foi significativa nas seguintes variáveis: sexo, nível de estresse, satisfação com a vida e consumo de álcool.

Ressaltamos que o ambiente universitário é marcado pela autonomia dos alunos e, em virtude disso, esse público deve ser monitorado nos aspectos relativos à saúde e à educação, juntamente com as condições que são ofertadas durante esse período acadêmico para que possam desenvolver o conhecimento teórico e prático sobre saúde.

\section{AGRADECIMENTOS}

Autores agradecem à Universidade Estadual do Ceará (UECE). Ao Grupo de Pesquisa Epidemiologia, Cuidado em Cronicidade e Enfermagem (GRUPECCE). À Coordenação de Aperfeiçoamento de Pessoal de Nível Superior (CAPES).

\section{REFERÊNCIAS}

1. Instituto Nacional de Estudos e Pesquisas Educacionais Anísio Teixeira. Microdados do Censo da Educação Superior, Brasília[Internet] 2017 [acesso 2018 Nov 16]; Disponível em: http://download.inep.gov.br/educacao_superior/ censo_superior/documentos/2018/censo_da_educacao_superior_2017notas_estatisticas2.pdf

2. Ojeda MAS, Bertos EDL. Healthy lifestyles of the university population. Nutr Hosp[Internet]. 2015 May [acesso 2015 Nov 16]; 1;31(5): 1910-9. Disponível em: https://www.ncbi.nlm.nih.gov/pubmed/25929358.

3. Almutairi KM, Alonazi WB, Vinluan JM, Almigbal TH, Batais MA, Alodhayani $A A$, et al. Health promoting lifestyle of university students in Saudi Arabia: a cross-sectional assessment. BMC Public Health[Internet]. 2018 [acesso 2018 Nov 16]; 18; 1093. Disponível em: https://bmcpublichealth.biomedcentral. com.ez76.periodicos.capes.gov.br/track/pdf/10.1186/s12889-018-5999-z. doi: 10.1186/s12889-018-5999-z.

4. Leite ACB, Grillo LP, Caleffi F, Mariath AB, Stuker H. Qualidade de vida e condições de saúde de acadêmicos de nutrição. Rev. Saúde Pública Paraná[Internet]. 2011 [acesso 2019 Jul 25]; 13 (1): 82-90. Disponível em: http://www.uel.br/revistas//uel/index.php/index/index.

5. Ozkan A, Turkmen M, Bozkus T, Kul M, Soslu R, Yasarturk F, Aydin R, et al. Determination of the Relationship between Healthy Lifestyle Behaviors, Physical Fitness, and Risk Factors of Coronary Heart Diseases in University Students. Educ. Sci[Internet]. 2018 Apr [acesso 2018 Nov 17]; 8: 51. Disponível em: file://C:/Users/Usuario/Downloads/education-08-00051.pdf.

6. Israa Assaf I, Brieteh F, Tfaily M, El-Baida M, Kadry S, Balusamy B. Students university healthy lifestyle practice: quantitative analysis. H. Inf. S. Systems[Internet]. 2019 [acesso 2019 Jul 25]: 7(1): 7. Disponível em: https:// link.springer.com/article/10.1007\%2Fs13755-019-0068-2.

7. Jaber R, Madhivanan P, Khader Y, Mzayek F, Ward KD, Maziak W. Predictors of waterpipe smoking progression among youth in Irbid, Jordan: A longitudinal study (2008-2011). Drug Alcohol Depend. [Internet]. 2015 Ago [acesso 2018 Dez 10]; 1(153): 265-70. doi: 10.1016/j.drugalcdep.2015.05.008.Disponível em: https:// www.sciencedirect.com/science/article/pii/S0376871615002446?via\%3Dihub.

8. Samet JM. Tobacco smoking: the leading cause of preventable disease worldwide. Thorac Surg Clin[Internet]. 2013 May [acesso 2018 Set 15]; 23(3):103-112. doi: 10.1016/j.thorsurg.2013.01.009. Disponível em: https:// www.thoracic.theclinics.com/article/S1547-4127(13)00010-8/fulltext

9. Tercyak KP, Rodriguez D, Audrain-McGovern J. High school seniors' smoking initiation and progression 1 year after graduation. Am J Public Health[Internet]. 2007 [acesso 2018 Set 15]; 97(8): 1397-1398. doi: 10.2105/AJPH.2006.094235. Disponível em: https://www.ncbi.nlm.nih.gov/pmc/articles/PMC1931449/.

10. Pimentel MH, Mata MA, Anes EM. Tabaco e álcool em estudantes: mudanças decorrentes do ingresso no ensino superior. Rev. Psicol Saúde Doenças[Internet]. 2013 [acesso 2018 Set 20]; 14(1): 12-18. Disponível em: http://www.scielo.mec. pt/scielo.php?script=sci_arttext\&pid=S1645-00862013000100012.

11. Meda SA, Gueorguieva RV, Pittman B, Rosen RR, Aslanzadeh F, Tennen $\mathrm{H}$, et al. Longitudinal influence of alcohol and marijuana use on academic performance in college students. PLOS ONE[Internet]. 2017 [acesso $2018 \mathrm{Dez}$ 13]; 12(3): 1-16. doi: 10.1371/journal.pone.0172213. Disponível em: https:// journals. plos.org/plosone/article?id=10.1371/journal. pone.0172213.

12. Sousa TF, Fonseca SA, José HPM, Nahas MV. Validade e reprodutibilidade do questionário Indicadores de Saúde e Qualidade de Vida de Acadêmicos (Isaq-A). Arq. Cienc Esporte[Internet]. 2013 [acesso 2018 Jul 22]; 1(1): 10-22. Disponível em: http://seer.uftm.edu.br/revistaeletronica/index.php/aces/ article/view/254.

13. Arsandaux J, Michel G, Tournier M, Christophe T, Cédric G. Is self-esteem associated with self-rated health among French college students? A longitudinal 
epidemiological study: the i-Share cohort. BMJ Open[Internet]. 2019 [acesso 2019 Jul 25]; 9: (024500). Disponível em: https://bmjopen.bmj.com/ content/9/6/e024500.

14. Ponte AV, Fonseca SCF, Carvalho MIMM, Fonseca JJA. Autoimagem Corpora e Prevalência de Sobrepeso e Obesidade em Estudantes Universitários. Rev Bras Promoç Saúde[Internet]. 2019 [acesso 2019 Jul 23]; 32(8510): 1-11. doi: http:// dx.doi.org/10.5020/18061230.2019.8510. Disponível em: https://periodicos. unifor.br/RBPS/article/view/8510/pdf.

15. Chow SKY, Lam KM, Lie SH, Mak KC, Mong KC, So CM, et al. Do demographic factors and a health-promoting lifestyle influence the self-rated health of college nursing students? BMC Nursing[Internet]. 2018 Nov [acesso 2019 Jul 25]: 17(50): 1-9. Disponível em: https://bmcnurs.biomedcentral.com/ articles/10.1186/s12912-018-0322-y.

16. Graner KM, Cerqueira ATAR. Revisão integrativa: sofrimento psíquico em estudantes universitários e fatores associados. Cienc. saúde coletiva[Internet]. 2919 Maio [acesso 2019 Jul 25]; 24(4): 1327-1346. Disponível em: http://www. scielo.br/scielo.php?script=sci_arttext\&pid=\$1413-81232019000401327.

17. Costa SMC; Monteiro PMF; Araújo JL; Rego ANF, Nascimento EGC. Consumo de Álcool entre Universitários do Interior do Nordeste Brasileiro. Rev. Aten. Saúde[Internet]. 2019 [acesso 2019 Jul 24]; 17(59): 88-94. doi: https://doi. org/10.13037/ras.vol17n59.5837. Disponível em: http://seer.uscs.edu.br/ index.php/revista_ciencias_saude/article/view/5837/pdf.

18. Barros MSMR, Costa LS. Perfil do consumo de álcool entre estudantes universitários. SMAD, Rev. Eletrônica Saúde Mental Álcool Drog[Internet]. 2019 [acesso 2019 Jul 24]; 15(1): 4-13. doi: http://dx.doi.org/10.11606/issn.18066976.smad.2019.000353. Disponível em: http://pepsic.bvsalud.org/scielo. php?script=sci_arttext\&pid=S1806- $9762019000100002 \& \operatorname{lng}=$ pt\&nrm=iso.

19. Laranjeira R, Pinsky I, Zaleski M, Caetano R, Secretaria Nacional Antidrogas. I Levantamento nacional sobre os padrões de consumo de álcool na população brasileira. CEBRID/UNIFESP[Internet]. Brasília: CISA, 2007 [acesso 2019 Jul 24]. p. 8-76. Disponível em: http://www.cisa.org.br/artigo/155/i-levantamentonacional-sobre-os-padroes.php.

20. Onetti-Onetti W, Minguet JLC, Martina FML, Rodriguez AF. Self-Concept and Physical Activity: Differences Between High School and University Students in Spain and Portugal. Front Psychol[Internet]. 2019 Jun [acesso 2019 Jul 23]; 20(10): 1333. doi: 10.3389/fpsyg.2019.01333. Disponível em: https://www. frontiersin.org/articles/10.3389/fpsyg.2019.01333/full. 